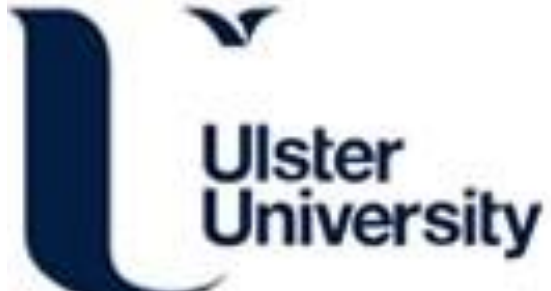

\section{Context-aware Characterisation of Energy Consumption in Data Centres}

Peoples, C., Parr, G., \& McClean, S. (2011). Context-aware Characterisation of Energy Consumption in Data Centres. In Unknown Host Publication (pp. 1250-1257). IEEE. https://doi.org/10.1109/INM.2011.5990573

Link to publication record in Ulster University Research Portal

\author{
Published in: \\ Unknown Host Publication
}

Publication Status:

Published (in print/issue): 18/08/2011

DOI:

10.1109/INM.2011.5990573

\section{Document Version}

Publisher's PDF, also known as Version of record

\section{General rights}

Copyright for the publications made accessible via Ulster University's Research Portal is retained by the author(s) and / or other copyright owners and it is a condition of accessing these publications that users recognise and abide by the legal requirements associated with these rights.

\section{Take down policy}

The Research Portal is Ulster University's institutional repository that provides access to Ulster's research outputs. Every effort has been made to ensure that content in the Research Portal does not infringe any person's rights, or applicable UK laws. If you discover content in the Research Portal that you believe breaches copyright or violates any law, please contact pure-support@ulster.ac.uk. 


\title{
Context-Aware Characterisation of Energy Consumption in Data Centres
}

\author{
C. Peoples, G. Parr and S. McClean \\ India-UK Advanced Technology Centre of Excellence in Next Generation Networks, Systems and Services \\ Faculty of Computing and Engineering, University of Ulster \\ Coleraine, Northern Ireland, United Kingdom \\ (c.peoples, gp.parr, si.mcclean)@ulster.ac.uk
}

\begin{abstract}
Carbon emissions are receiving increased attention and scrutiny in all walks of life and the ICT sector is no exception. With the increase in on-demand applications and services together with on-demand compute/storage facilities in server farms or data centres there are self-evident increases in the power requirements to maintain such systems. Proponents of the impact of increased carbon emissions when powering electrical systems in general however, regularly impress negative side-effects such as influence on climate change. Action is subsequently being encouraged to halt further environmental damage. The problem is explored in this paper from the point of view of carbon emissions from data centre operations and the development of energy-aware management and energy-efficient networking solutions. Data centre energy consumption costs drive the evaluation process within a Data Centre EnergyEfficient Context-Aware Broker (DCe-CAB) algorithm designed as an original solution to this significant carbon-contributing network scenario. In this paper, performance requirements and objectives of the DCe-CAB are defined, along with case study demonstration of the way in which it optimises selection and operation of data centres using context-awareness.
\end{abstract}

Keywords-carbon cost, context-awareness, data centre, energyaware management, energy-tolerant networking, 'green' IT.

\section{INTRODUCTION}

Data centres are expanding in both size and number in response to an ever growing volume of user generated content and Internet-based distribution of software components for services and applications. Defining the data centre market size and organisation accurately however, is difficult due to commercial secrecy associated with the number of plants, hardware used and methods of operation within, a fact which companies believe protects against attention concerned about their contributions to the carbon footprint. Estimations of the size and number of data centres nonetheless prompt concerns in relation to their carbon footprint [1]: the Code of Conduct on Data Centre Energy Efficiency states that electricity consumption in Western Europe was 56 TWh in 2007 and is estimated to increase to $104 \mathrm{TWh}$ by 2020 [2]. Telecom equipment across the data centre-access-metro-core networks

This research is supported by the India-UK Advanced Technology Centre of Excellence in Next Generation Networks (IU-ATC), funded by the UK EPSRC Digital Economy Programme and Government of India Department of Science and Technology (DST). path therefore plays a role in its contribution to carbon emissions (assuming a non-renewable energy mix at the electricity provider). As data centres are an integral part of the telecommunication market across the end-to-end path with clients, some data centre operators are becoming more explicit with respect to their operational strategy in an attempt to demonstrate social and environmental responsibility and prevent negative attention which may restrict their future growth. Many large organisations, for example, promote the 'green' schemes deployed in association with their buildings and/or internal operations. Each solution is however, in general company-specific [3]-[5]. Due to increasing concerns associated with data centre emissions, standardised approaches which allow greater efficiency here are anticipated. Data centre management frameworks identified in the literature however, concentrate on operation within the DC without considering the management of communications on the end-to-end path with corporate or enterprise clients and eventually end-consumers of the content or service. This is therefore the focus of our research framework.

Several characteristics may be considered when managing data centres for energy-efficiency purposes, including their size, server capabilities, location in relation to clients, renewable energy supply and power effectiveness. The solution presented in this paper is driven by energy consumption costs of data centres and on paths with clients (Fig. 1): cost contributors between and at these end points are evaluated within a Data Centre (DC) Energy-Efficient Context-Aware Broker (e-CAB) algorithm. (Electricity cost is also considered due to sustainability objectives for operators as a further aim of

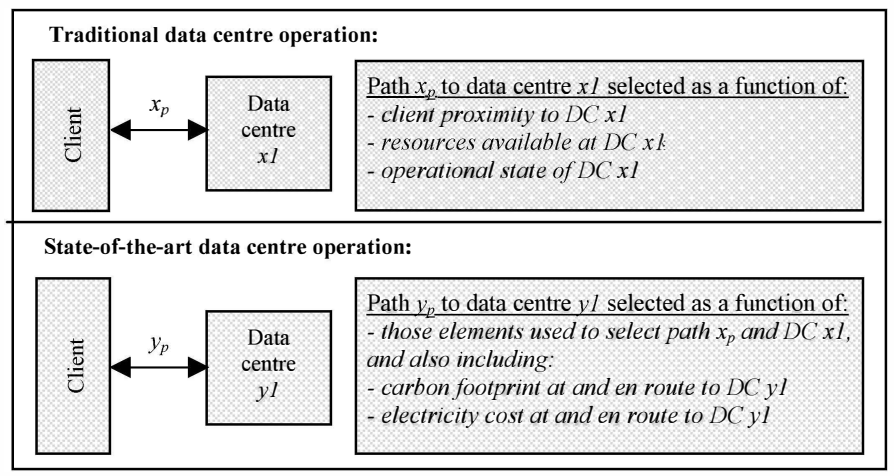

Figure 1. Modernisation of data centre operation 
improved efficiency.) The DCe-CAB explores context in realtime to enforce intelligent decision-making in data centres and on links with clients. In this paper, operational objectives of the DCe-CAB are defined mathematically along with demonstration of the effectiveness of optimised selections in a case study scenario.

This paper continues as follows: in Section II, several 'green' strategies adopted by DC operators and from the research community are discussed to highlight the research gap which exists. The DCe-CAB algorithm is presented in Section III in response. Context attributes used in its management and optimisation algorithm are explored in Section IV and after some case study results are discussed, the paper concludes and presents future work in Section V.

\section{RELATED WORK}

Energy-efficient operational strategies in data centres are influenced by efforts from govermmental bodies. The Code of Conduct on Data Centres Energy Efficiency applies to both IT equipment within data centres and facilities which provide an optimum operating environment, and includes definition of metrics which indicate data centre energy-efficiency, implementation of monitoring action to measure their success and identification of areas where improvements are possible [2]. However, while businesses (including data centres) are taxed on carbon contributions according to the Climate Change Levy, a fixed strategy on emission reduction does not exist. Furthermore, compliance with the Code is voluntary and companies are not obliged to follow recommendations. Application of strategies in this field therefore remains open from the point of view of government regulation.

The independent research community is also involved in developing energy-efficient data centre solutions. Patterson (2008) defines a model to determine the optimum temperature to operate data centres, calculated as a function of the equipment, architecture of the cooling system and location of the data centre [6]. They experiment with increasing the temperature and measuring effects on the chiller system and Power Usage Effectiveness (PUE). Nathuji et al. (2007) propose another solution for the data centre to control traffic loading as a function of platforms on which network resources exist: platforms where greatest power savings can be achieved are selected by the management system [7]. Parolini et al. (2008) suggest a data centre management strategy which controls cooling and computational operation simultaneously [8]. This solution exploits the fact that cooling is applied in DCs in response to hardware use and their management in relation to each other can therefore optimise operational efficiency. Taking these contrasting strategies into account, proposals from the literature demonstrate a range of approaches to optimising energy-efficiency in data centres.

A review of related literature however, also indicates that solutions are incomplete; an intelligent DC configuration strategy is proposed in [6], for example, but without consideration for management of external context to allow a complete DC solution. Support of such systems requires that context for aspects which may be managed in data centres has been collected. This gap is filled by recent 'work in progress'
Internet Drafts which define Management Information Base (MIB) structures for energy-efficiency purposes in networks in general [9]-[10]. As with related literature however, they manage aspects of network operation, but not the complete range of scenarios which may be encountered.

To extend the contribution of these standalone approaches, they can be housed within more thorough network management frameworks. Examples have been identified in the literature: Autopilot from Microsoft provisions functionalities for complete DC operation and management, from monitoring to enabling service and recommending hardware replacement. DCell from Guo et al. (2008) provides a contrasting management function in DCs, performing distributed fault tolerant routing and a scaling strategy is invoked in the instance that data centre resources expand [11]. These examples therefore validate that management frameworks for a greater range of DC operational issues exist.

We however, consider the DC energy-efficiency aspect further by identifying that although individual approaches to improving energy-efficiency are housed within management frameworks and can accommodate a range of operational challenges in data centres, they fail to consider end-to-end paths from clients to the point of service. A research gap in current solutions therefore exists, and we provide an original solution in the DCe-CAB which collects, manages, and evaluates context from the environment, data centre and applications, and possesses capability to manage aspects within the DC, including communication with clients. Inclusion of the end-to-end path in decisions made relates to DC resource selection as a function of client proximity and ability to support application Service Level Agreements (SLA) (Fig. 1). This notion is extended with detail regarding the number of and performance achieved at intermediary nodes traversed on the end-to-end path between client device and data centre and the inherent carbon and financial cost incurred. The overall intention is therefore to provision a solution where cost on the path is also evaluated as a QoS parameter. In filling this research gap we take competitive work forward by exploring MIB attributes collected for energy-efficiency purposes which will be used to define policies for enforcement of energy-aware decisions in DCs and in the brokering function with clients.

\section{RESEARCH APPROACH: DATA CENTRE E-CAB}

The Context-Aware Broker (CAB) architecture was developed by the authors originally to overcome reliability and sustainability challenges in Delay-Tolerant Networks (DTNs) [12]. Taking into account the need to reduce IT-driven carbon emissions specific to the data centre problem, the DCe-CAB optimises communication processes in parallel with the original CAB strategy due to severe resource constraints in deep space while also considering the energy-constrained conditions here. Where resources are restricted and/or the objective is to minimise energy consumption, network management strategies aim to minimise overall cost per transmission. There is therefore an overlap between use of the $\mathrm{CAB}$ architecture in delay- and energy-tolerant networks (ETNs). Applied instead of a generic management platform, the DCe-CAB collects context data on the domain within which it is applied and executes optimisations to maximise performance in a structured 


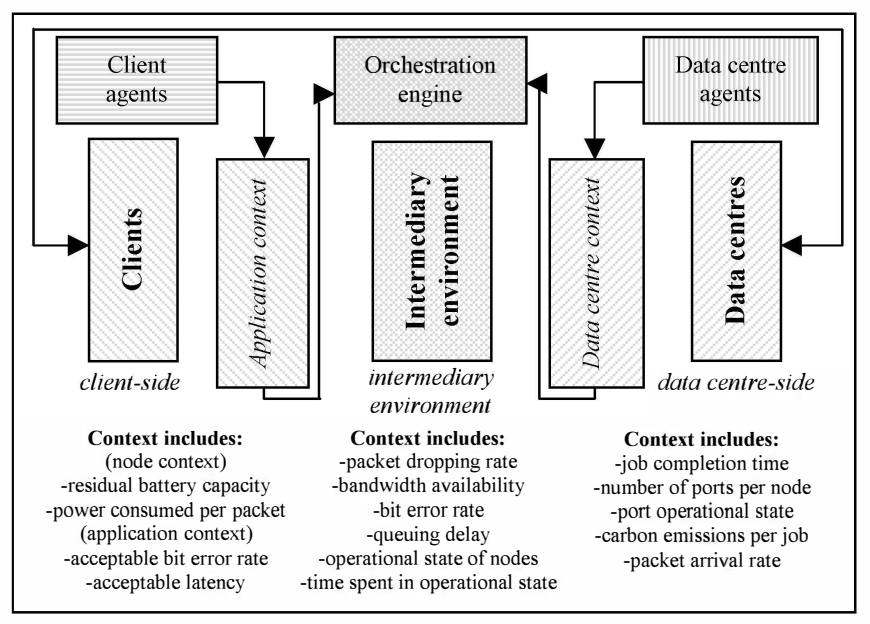

Figure 2. DCe-CAB positioning in the data centre network

approach that takes network constraints into account.

DCe-CAB placement when integrated into the data centre network is represented in Fig. 2. Alongside each client, an application DCe-CAB agent invokes collection of application Quality of Service (QoS) context attributes. In parallel, a data centre e-CAB agent controls the invocation of context detail from each DC component. Clients communicate across the network core to the selected data centre as instructed by the $D C e-C A B$ Orchestration engine, a management system which controls a range of centres and devices and operates in parallel with existing DC brokering systems such as VMWare vSphere and Citrix solutions. The Orchestration engine's functionality in relation to application and DC agents when performing endto-end management is represented in Fig. 3. Once a transmission request is communicated (step 1 in Fig. 3), the Orchestration engine controls simultaneous collection of context (cxt) from client and DC sides (step 2), before feeding it through the evaluation process (step 3). Real-time ability to achieve QoS while optimising energy-efficiency within network and data centre operational constraints is determined; the network and data centre state definition is made (step 4) and management decisions involving selection of a data centre and path are defined on a hop-by-hop and end-to-end approach (step 5): decisions are made by the Orchestration engine as a function of client location, average packet throughput, energy consumption and job completion rate. Once transmission begins, a range of attributes are monitored (step 6) to optimise the DCe-CAB's dynamic ability to maintain energy-awareness and efficiency. The DCe-CAB may also predict, using historical data retained and past network trends identified, future performance. It may anticipate, for example, that throughput will continue at the same rate and acknowledge that energy consumption levels at a DC will exceed a threshold. Recovery action in preparation for this event may therefore avoid the negative effects associated with its occurrence. Alarms once transmission begins (step 7) can protect against exposure to network events with negative consequences. An alarm may indicate, for example, that, given the Bit Error Rate (BER) and reliable communication scheme used, job completion time at a DC is increasing. Action can subsequently be invoked to optimise energy consumption in relation to network constraints and application requirements. This can

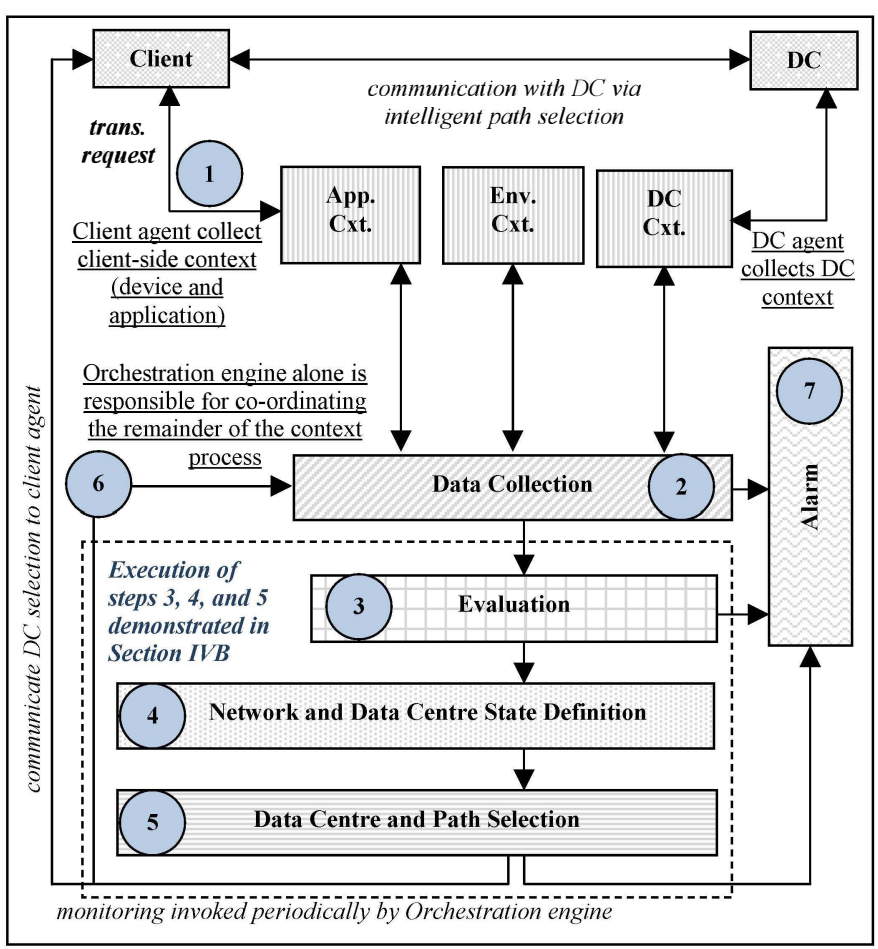

Figure 3. DCe-CAB Orchestration engine states

involve re-selection of the path to the $\mathrm{DC}$, re-selecting the $\mathrm{DC}$ to one with lower power costs, suspending transmission until network conditions improve or re-starting transmission.

While the architecture developed for DTNs [12] in general is being reused, algorithms within its core phases have been adapted for the DC domain, including context collection, monitoring procedures invoked, evaluations involved and actions applied. In the DC domain there is, for example, a degree of redundancy not found in the deep space domain. There will therefore be more opportunities to send ports to 'sleep' as a function of throughput. In deep space by way of contrast, sending nodes to 'sleep' is a more critical action, and decisions will be based on pre-planned maintenance strategies in accordance with intended node position at a point in time. Extensions to the original $\mathrm{CAB}$ algorithm therefore exploit its capabilities and fast-track the development process.

Over-riding all decisions made by the DCe-CAB is the objective of maintaining a positive cost-benefit balance. The DCe-CAB has therefore been tailored to optimise additional energy requirements introduced by increased decision-making: real-time decisions are made, therefore, to optimise the volume of context data collected during refreshes and rate at which monitoring occurs. In an optimum configuration, only those attributes which may affect the communication will be monitored. These tailoring decisions maintain a solution which is itself energy-aware.

\section{Mathematical Modelling OF DCe-CAB Optimisation}

Context attributes drive operation of the DCe-CAB by influencing policy invocation. A range of energy-saving strategies, decision variables, objective functions and operational constraints are defined in the following section to 
demonstrate the conditions within which the DCe-CAB achieves optimisation. In addition to those in Fig. 2, context attributes used by the DCe-CAB include:

- Application requirements: Transmission volume, QoS;

- Network: Bandwidth, Bit error rate, Queuing delay, Throughput, Packet loss ratio, Number of path nodes;

- Data centre: Energy metric, Task waiting time, I/O rack operations, I/O server operations, Electricity unit cost;

- Client: Propagation delay to data centre;

- Environment: Maximum power cost per unit at any DC under the DCe-CAB's control; Maximum energy emissions from any DC under the DCe-CAB's control.

Power consumption at the data centre is specified in terms of an energy metric as opposed to a carbon metric. It is assumed that the higher the energy metric value, the greater the carbon emission; we recognise however, that it could vary based on the energy mix of the electricity provider - a DC may consume a large amount of energy, but emit almost no $\mathrm{CO}_{2}$ due to its intentional location close to a renewable energy power plant. Application QoS is defined in terms of the required bandwidth, acceptable bit error rate, latency and packet loss ratio. Mathematical models described in this section are based on [13], in which similar models to reconfigure networks in the instance of link failure are developed. We take this work forward by applying it to data centres and using new context attributes relevant in this domain.

\section{A. Mathematical Modelling:}

\section{1) Principles of the DC Scenario and DCe-CAB Objectives}

Let us consider a network as a graph $G=(V, E)$ composed of a set $V:=V(G)$ of nodes and $E:=E(G)$ of links. Let us also consider a data centre as a graph $H=(N, L)$ composed of a set $N:=N(H)$ of nodes and $L:=L(H)$ of links. A path $p$ to the data centre, $p=(v, i, j, \ldots, k, h)$, is composed of a series of subpaths $(v, i),(i, j), \ldots,(k, h)$ between source $v$ and destination $h$. $p_{i, j}=\left(v_{i}, \ldots, v_{j}\right)$ represents all sub-paths of this path. A path $p i$ within the data centre, $p i=(m, n, o, \ldots, q, s)$, is composed of a series of links $(m, n),(n, o), \ldots,(q, s)$ between the point of entry to data centre at $m$ and destination server $s$. $p i_{n, o}=\left(v_{n}, \ldots, v_{o}\right)$ represents all sub-paths of this path. A data centre $D C=\left(s_{1}, r c_{1}, \ldots, s_{c}, r c_{c}\right)$ is composed of nodes which may represent a rack $r c$ containing multiple servers or an individual server $s$. Elements $\left(s_{1}, r c_{1}, \ldots, s_{c}, r c_{c}\right)$ are nodes of $D C$. Carbon emissions from data centre $D C$ will be a sum of the maximum volume of emissions from each device, summed as server $s$ and/or rack $r c$ emissions from the total number $c$ of rack and server devices on path $p i$ within data centre network $H$. Emissions from each device will vary as a function of delay in the network: the maximum task waiting time $l$ between client request and response is infinity.

Each transmission leaving the client can be characterised with a weight indicating the priority of minimising energy consumption or task waiting time (power conservation will not occur at the expense of application QoS). This is communicated to the DCe-CAB Orchestration engine during execution of step 1 in Fig. 3. The objectives defined in equations (1) to (4) may be applied to jobs leaving the client (mathematical symbols used are also defined in Table I):

- Minimising energy consumption en of jobs serviced within the data centre, objective $M_{e n}$ :

$$
\text { minimise } \underset{p i \in P^{*(n d ; H)}}{e n}, \text { minimise } \underset{p i \in P^{*(n, o ; H)}}{e n}
$$

This involves minimising energy consumption en when operating nodes $n d$ within data centre network $H$ on path $p i$ where $P^{*(n d ; H)}$ is the set of all nodes traversed on path $p i$ in network $H$ and the cost of operating sub-links on path $p i$ where $P^{*(n, o ; H)}$ is the set of all sub-links traversed between end points $(n, o)$ in data centre network $H$.

- Minimising energy consumption $c c$ on links between client and data centre, objective $M_{c c}$ :

$$
\text { minimise } \underset{p \in P^{*}(i, j ; G)}{c c}
$$

This involves minimising the total carbon cost $c c$ of operating on path $p$ where $P^{*(i, j ; G)}$ is the set of all sub-links traversed between end points $(i, j)$ in network $G$.

- Minimising electricity cost $e l$ at the DC, objective $M_{e l}$ :

$$
\text { minimise } \underset{p i \in P^{*}(n d ; H)}{e l}
$$

This involves minimising the energy consumption el when operating nodes $n d$ within data centre network $H$ on path $p i$ where $P^{*(n d ; H)}$ is the set of all nodes traversed on the end-to-end path in network $H$.

- Minimising latency $l$ between client request and response, objective $M_{l}$ :

$$
\begin{aligned}
& \text { minimise } \underset{p i \in P^{*(n d ; H)}}{l} \text {, minimise } \underset{p \in P^{*(n d ; G)}}{l} \\
& \text { minimise } l_{p i \in P^{*(n, o ; H)}}, \text { minimise } l_{p \in P^{*(i, j ; G)}}
\end{aligned}
$$

This involves minimising the latency incurred in network $G$ on path $p$ between end points $(i, j)$, on the end-to-end path $p i$ between end points $(n, o)$ within data centre network $H$, and at nodes $n d$ in DC network $H$ and external network $G$.

These objectives prioritise either selection of a DC or path to the DC as a function of carbon cost, electrical cost or job completion latency. In this evaluation, we assume that applications have full accuracy and reliability requirements, and that the achievement of QoS is measured solely by job completion latency. Effective path selection will be important where the characteristics which distinguish between data centre capabilities are similar, there are a range of DCs from which 
Table I.

MATHEMATICAL SyMBOLS AND DEFINITIONS

\begin{tabular}{|c|c|c|c|}
\hline Symbol & Definition & Symbol & Definition \\
\hline$c c$ & $\begin{array}{l}\text { energy consumption on } \\
\text { sub-links between } \\
\text { client and DC }\end{array}$ & $p i$ & $\begin{array}{l}\text { path within DC from } \\
\text { point of entry to point of } \\
\text { service in network } H\end{array}$ \\
\hline$E$ & $\begin{array}{l}\text { set of sub-links in } \\
\text { network external to DC }\end{array}$ & $p_{\in} P^{*(i, j ; G)}$ & $\begin{array}{l}\text { set of all sub-links } \\
\text { traversed between end } \\
\text { points }(i, j) \text { on path } p \text { in } \\
\text { network } G\end{array}$ \\
\hline el & $\begin{array}{l}\text { electricity cost per unit } \\
\text { at DC }\end{array}$ & $p i_{\in} P^{*(n d ; H)}$ & $\begin{array}{l}\text { set of all nodes } n d \\
\text { traversed on path } p i \text { in } \\
\text { network } H\end{array}$ \\
\hline$V$ & $\begin{array}{l}\text { set of nodes in network } \\
\text { external to DC }\end{array}$ & $p_{\in} \in P^{*(D C ; G)}$ & $\begin{array}{l}\text { data centre(s) } D C \text { in } \\
\text { network } G \text { on path } p \\
\text { from client device }\end{array}$ \\
\hline en & $\begin{array}{l}\text { energy consumption at } \\
\text { DC }\end{array}$ & $p_{\in} P^{*(G)}$ & $\begin{array}{l}\text { path } p \text { traversed within } \\
\text { network } G\end{array}$ \\
\hline$G$ & $\begin{array}{l}\text { network external to DC } \\
\text { (between client and } \\
\text { point of entry to DC) }\end{array}$ & $r c$ & DC rack device \\
\hline$H$ & internal DC network & $s$ & DC server device \\
\hline$i$ & $\begin{array}{l}\text { client device in } \\
\text { network } G\end{array}$ & $x$ & traffic flow \\
\hline$j$ & $\begin{array}{l}\text { point of entry to DC in } \\
\text { network } G\end{array}$ & $\left(x_{p}\right)_{p \in P^{*}(G)}$ & $\begin{array}{l}\text { volume of traffic } x \\
\text { traffic transmitted across } \\
\text { path } p \text { in network } G\end{array}$ \\
\hline$i, j$ & $\begin{array}{l}\text { communicating end } \\
\text { points on path } p \text { (which } \\
\text { may include sub-links } \\
\text { in between) in network } \\
\text { external to DC }\end{array}$ & $x_{p}^{c c}$ & $\begin{array}{l}\text { carbon cost associated } \\
\text { with flow } x \text { arriving on } \\
\text { path } p \text { at DC }\end{array}$ \\
\hline$L$ & $\begin{array}{l}\text { set of sub-links within } \\
\text { DC }\end{array}$ & $x_{p}^{e l}$ & $\begin{array}{l}\text { electricity cost incurred } \\
\text { by flow } x \text { arriving on } \\
\text { path } p \text { at DC }\end{array}$ \\
\hline$l$ & $\begin{array}{l}\text { latency between client } \\
\text { request and response } \\
\text { from DC }\end{array}$ & $x_{p}^{\text {elThreshold }}$ & $\begin{array}{l}\text { threshold electricity cost } \\
\text { which may be incurred } \\
\text { by flow } x \text { on path } p\end{array}$ \\
\hline $\bar{N}$ & $\begin{array}{l}\text { set of nodes within } \\
\text { internal DC network }\end{array}$ & $x_{p i}^{e n}$ & $\begin{array}{l}\text { carbon cost associated } \\
\text { with flow } x \text { within DC } \\
\text { network on path } p i\end{array}$ \\
\hline$n d$ & node & $x_{p}^{l}$ & $\begin{array}{l}\text { latency cost associated } \\
\text { with flow } x \text { on path } p\end{array}$ \\
\hline$n, o$ & $\begin{array}{l}\text { communicating end } \\
\text { points (which may } \\
\text { include sub-links in } \\
\text { between) in internal } \\
\text { DC network } H\end{array}$ & $x_{p}^{l \text { lThreshold }}$ & $\begin{array}{l}\text { threshold latency cost } \\
\text { which may be incurred } \\
\text { by flow } x \text { on path } p\end{array}$ \\
\hline$p$ & $\begin{array}{l}\text { path to DC from client } \\
\text { device in network } G\end{array}$ & $x_{p}^{n d}$ & $\begin{array}{l}\text { number of nodes } \\
\text { traversed by flow } x \\
\text { arriving at DC on path } p\end{array}$ \\
\hline
\end{tabular}

service could be requested and the path traversed is the limiting factor on cost and/or latency. Effective DC selection on the other hand will be important where power effectiveness in those data centres closest to the client varies between centres. Operational performance will therefore be optimised when selections are made using these characteristics.

\section{2) Solving Competing Optimisation Objectives}

Achieving this multi-objective problem (defined in equations (1) to (4)) simultaneously however, presents a constrained optimisation challenge. It may not be possible, for example, to minimise en and $l$ in parallel: when $e n$ is reduced, an increase in $l$ due to reduced throughput and/or increased congestion may be more likely. In minimising a single attribute, the others will suffer as a result. The highest level of performance will therefore be achieved by prioritising a single performance characteristic while maintaining other measurable attributes above a threshold. For the purpose of this research, objectives prioritise energy cost while incurring threshold latency and electrical costs. The overall objective in selecting a data centre, executed during step 5 in Fig. 3 is therefore to:

$$
\operatorname{minimise} \sum C\left(x_{p i}^{e n}+x_{p}^{c c}\right)
$$

allowing costs $C$ en (1) and $c c$ (2) associated with flow $x$ on path $p$ at and path $p i$ within a DC to be minimised, while maintaining average electricity cost $e l$ (3) and latency $l$ (4) incurred below a threshold $(e l$ in (6) may be replaced with $l$ ):

$$
x_{p}^{e l} \leq x_{p}^{e l T h r e s h o l}
$$

A further objective involves restricting the number of nodes $n d$ traversed by flow $x$ on path $p$ :

$$
x_{p}^{n d}
$$

These objectives are applied subject to application QoS requirements to optimise costs on all sub-links traversed. This will involve ensuring, for example, that the bandwidth available on each sub-link is greater than or equal to the application's requirements. Similar requirements may be specified to select a path with lowest error rate, packet loss ratio and throughput. These constraints are motivated by their positive impact on conserving power consumption: by restricting transmissions to those paths where operational conditions are best, this will help to prevent expiration of protocol time-to-live characteristics and reduce reliance on reliability mechanisms, thereby reducing the cost per transmission. CPU utilisation also impacts on power, but in this model, irrespective of operational characteristics such as this or DC power effectiveness, overall operation of the data centre network is represented by its carbon contribution. This explains the motivation of constraints including number of nodes on the path (each consuming energy), application transmission volume (influencing energy consumed at each node), bandwidth (influencing invocation of reliability mechanisms) and carbon emissions on the path to the DC as contributory factors on paths external and internal to the data centre.

This is a complex optimisation problem to solve, given its multi-objective combinatorial nature. These optimisations can be solved within the DCe-CAB Orchestration engine using branch and bound techniques by dividing the problem into subclasses for solving. In the DC domain, this includes the problem of minimising carbon cost incurred internally and externally to the DC, while simultaneously maintaining lower bounds on financial cost, latency and node number, such that a balance between competing parameters is achieved.

\section{B. Case Study}

An exemplar DCe-CAB execution of steps 3, 4, and 5 in Fig. 3 are considered using the network topology scenario presented in Fig. 4. An assumption in the management approach proposed for use in such a scenario is that DCs under the DCe-CAB's control are grouped on an operator- specific basis. The DCe-CAB could therefore be located within DC6 in the scenario in Fig. 4 when controlling operation of DCs 1 to 12 due to its central location and therefore average cost of 


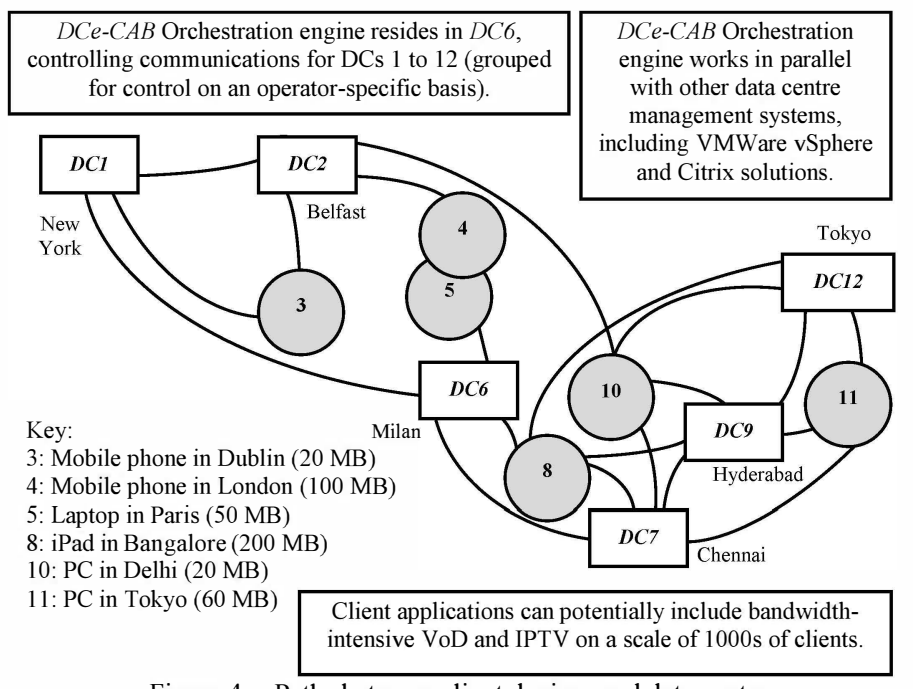

Figure 4. Paths between client devices and data centres

Table II. Network Link CAPACITIES (MEgabytes)

\begin{tabular}{|c|c|c|c|c|c|c|}
\hline Link ID & $\mathbf{a}$ & c & e & f & $\mathbf{h}$ & d \\
\hline Link & 1,2 & 1,3 & 2,3 & 2,4 & 5,6 & 6,1 \\
\hline Capacity (MB) & 500 & 210 & 300 & 440 & 1,200 & 700 \\
\hline Link ID & $\bar{i}$ & $\mathbf{j}$ & $\mathbf{u}$ & $\mathbf{s}$ & $\mathbf{r}$ & $t$ \\
\hline Link & 6,8 & $\frac{5,7}{6,7}$ & 7,8 & 7,10 & 7,9 & 8,9 \\
\hline Capacity (MB) & 600 & 800 & 850 & 150 & 100 & 300 \\
\hline Link ID & \multirow{2}{*}{\multicolumn{2}{|c|}{ I }} & $\mathbf{m}$ & $q$ & o & $\mathbf{n}$ \\
\hline Link & \multirow{2}{*}{\multicolumn{2}{|c|}{$\frac{\mathbf{1 0 , 1 2}}{400}$}} & 10,9 & 9,11 & 11,12 & 9,12 \\
\hline Capacity (MB) & & & 200 & 900 & 1,000 & 100 \\
\hline
\end{tabular}

Table III. DATA CEnTRE CARbon EMission CONVERSion FaCtors [14]

\begin{tabular}{|c|c|c|c|c|c|c|}
\hline DC ID & $\mathbf{1}$ & $\mathbf{2}$ & $\mathbf{6}$ & $\mathbf{7}$ & $\mathbf{9}$ & $\mathbf{1 2}$ \\
\hline $\begin{array}{c}\text { Carbon Emission } \\
\text { Conversion Factor }\end{array}$ & 0.1 & 0.2 & 0.3 & 0.15 & 0.25 & 0.5 \\
\hline
\end{tabular}

context collection and management in general. It is not the intention to suggest that this topology is representative of all DCs worldwide, nor that a single DCe-CAB will be responsible for influencing operation of all. Where operators adjust traffic within the scope of the SLAs to minimise their energy consumption, these adjustments will be applied consistently when DCs are controlled on an operator-specific basis.

\section{1) Network Characteristics}

The network in Fig. 4 can be characterised by its link capacities (Table II), operational efficiency of DCs (measured using a carbon conversion factor based on examples in [14] which indicates overall plant efficiency) (Table III) and link characteristics on paths between devices and DCs. Energy cost in data centres in general is calculated as a function of electricity consumption (measured in terawatt hours) which increases in parallel with workload. This is weighted by a conversion factor (measured in kilograms of $\mathrm{CO}_{2}$ emitted per kilowatt hour) to determine the overall carbon cost (measured in tonnes of $\mathrm{CO}_{2}$ emitted). In exploring $\mathrm{DC}$ cost using the conversion factor, we therefore consider plant efficiency when assuming a constant workload at each. In the scenarios under review in Fig. 4, there are instances where the client is connected directly to a DC and also where it is connected to DCs several hops away. Application traffic may therefore pass directly to or through multiple DCs to reach the DC which will service the request. Compounded by the range of efficiencies in the test scenario, the DCe-CAB's opportunity to perform intelligent selection in the exemplar scenario is highlighted. There are a range of network characteristics which can be captured and used in the decision-making process. Network link capacities are used because this characteristic influences path ability to support an application transmission. The number of intermediary path nodes is therefore also important when evaluated in relation to bandwidth availability: where bandwidth constraints force retransmissions, the number of intermediary nodes on the path will influence the overall cost to which a transaction is exposed.

\section{2) Autonomic Energy-Aware Data Centre Selection}

The objective of this research is to automate selection of a DC in response to an application request and operational characteristics of DCs and links between device and data centre. The processes described in this section are executed in the DCe-CAB's evaluation phase represented in Fig. 3 (step 3). DC selection can therefore be made intelligently by including a range of context attributes in the decision-making process:

1. Path selection as a function of the number of nodes nd in network $G$ traversed en route to the $D C$ on path $p$, transmission volume $x$ from the client device, minimum bandwidth available $B_{\min }$ between $D C$ and device, and average volume of carbon emissions en from DCs on the path between device and $D C(8)$ :

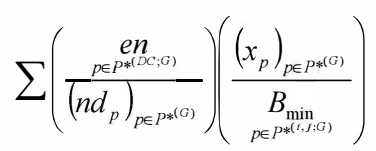

The objective of this evaluation is to select the path for which carbon emissions in network $G$ are minimised while maintaining a threshold level of application QoS. It is for this reason that the minimum link bandwidth $B_{\min }$ available on the end-to-end path $(i, j)$ is included in the equation, measured in relation to the volume of application traffic $x$ sent. The path with the minimum calculated value is selected as the optimum route when (8) is used.

Performance results for devices 3 (Table IV), 4 (Table V) and 5 (Table VI) when (8) is applied are shown. Path $c(3,1)$ is selected as the optimum for device $3, f, a(4,2,1)$ is the optimum for device 4 and $h(5,6)$ is the optimum for device 5 . Using $(8)$, the DCe-CAB strikes a balance in its selection between carbon emissions in relation to number of nodes, and transmission volume in relation to bandwidth. This is evident from the fact that it is not strictly the extreme (maximum or minimum)

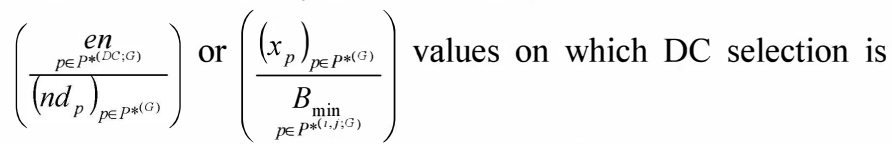
made in each case. The DCe-CAB will however, sacrifice a higher cost in the data centre and in terms of the number of nodes traversed to achieve a scenario where a greater volume of bandwidth is available on the path between communicating nodes in relation to the volume of traffic sent. 
Table IV. $\quad$ PATH SELECTION FROM DEVICE 3 USING (8)

\begin{tabular}{|c|c|c|c|c|c|c|c|c|c|}
\hline Client & \multicolumn{6}{|c|}{ Path to Data Centre } & \multirow{2}{*}{$\begin{array}{c}\left(\begin{array}{c}e n \\
\frac{p \in P^{(x) ;(G)}}{\left(n d_{p}\right)_{p \in P \times(G)}}\end{array}\right) \\
\mathbf{0 . 1}\end{array}$} & \multirow{2}{*}{ 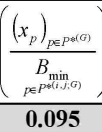 } & \multirow{2}{*}{$\begin{array}{c}\text { (8) } \\
\\
0.0095\end{array}$} \\
\hline 3 & 1 & - & - & - & - & - & & & \\
\hline 3 & 2 & - & - & - & - & - & 0.2 & 0.067 & 0.013 \\
\hline 3 & 1 & 6 & & - & - & - & 0.2 & 0.095 & 0.0119 \\
\hline 3 & 2 & 1 & - & - & - & - & 0.15 & 0.067 & 0.01 \\
\hline 3 & 2 & 1 & 6 & - & - & - & 0.2 & 0.067 & 0.013 \\
\hline 3 & 2 & 1 & 6 & 7 & - & - & 0.187 & 0.067 & 0.0125 \\
\hline 3 & 2 & 1 & 6 & 7 & 9 & - & 0.2 & 0.2 & 0.04 \\
\hline 3 & 2 & 1 & 6 & 7 & 9 & 12 & 0.25 & 0.2 & 0.05 \\
\hline
\end{tabular}

Table V.

PATH SELECTION FROM DEVICE 4 USING (8)

\begin{tabular}{|c|c|c|c|c|c|c|c|c|c|}
\hline Client & \multicolumn{6}{|c|}{ Path to Data Centre } & \multirow{2}{*}{ 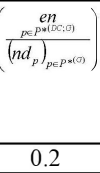 } & \multirow{2}{*}{$\frac{\left(\frac{\left(x_{p}\right)_{p \in p^{(G)}}}{B_{\min }}\right)}{0.227}$} & \multirow{2}{*}{\begin{tabular}{|c|} 
(8) \\
0.0455
\end{tabular}} \\
\hline 4 & 2 & - & - & - & - & - & & & \\
\hline 4 & 2 & 1 & - & - & - & - & 0.15 & 0.227 & 0.0341 \\
\hline 4 & 2 & 1 & 6 & - & - & - & 0.2 & 0.227 & 0.0455 \\
\hline 4 & 2 & 1 & 6 & 7 & - & - & 0.187 .5 & 0.227 & 0.0426 \\
\hline 4 & 2 & 1 & 6 & 7 & 9 & - & 0.2 & 1 & 0.2 \\
\hline 4 & 2 & 1 & 6 & 7 & 9 & 12 & 0.25 & 1 & 0.25 \\
\hline
\end{tabular}

Table VI.

PATh Selection From DeVice 5 USING (8)

\begin{tabular}{|c|c|c|c|c|c|c|c|}
\hline Client & \multicolumn{4}{|c|}{ Path to Data Centre } & \multirow{2}{*}{ 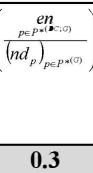 } & \multirow{2}{*}{ 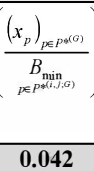 } & \multirow{2}{*}{$\begin{array}{c}\text { (8) } \\
\\
0.0125\end{array}$} \\
\hline 5 & 6 & - & - & - & & & \\
\hline 5 & 6 & 1 & - & - & 0.2 & 0.071 & 0.0143 \\
\hline 5 & 6 & 7 & - & - & 0.225 & 0.063 & 0.0140 \\
\hline 5 & 6 & 1 & 2 & - & 0.2 & 0.1 & 0.02 \\
\hline 5 & 6 & 7 & 9 & - & 0.233 & 0.5 & 0.1167 \\
\hline 5 & 6 & 7 & 9 & 12 & 0.3 & 0.5 & 0.15 \\
\hline
\end{tabular}

A DC may also be selected based on the relationship between different context attributes:

2. Path selection as a function of cost in the selected data centre en, traffic volume $x$, path cost cc across all sub-links on the end-to-end path in network $G$ between client and $D C$, and number of nodes nd traversed by the transmission on path $p(9)$ :

$$
\left(\left(x_{p}\right)_{p \in P^{*(G)}} \underset{p \in P *(D C ; G)}{e n}\right)\left(\underset{p \in P^{*(i, j ; G)}}{c \mathcal{C}}\left(n d_{p}\right)_{p \in P *(G)}\right)
$$

This calculation relates energy consumption in a DC to the volume of traffic passing in, and average carbon costs from intermediary nodes and links traversed en route to the DC in relation to the number between source and destination. The minimum value calculated is selected as the optimum route.

Data and calculations used in the selection of a DC using (9) for devices 3 (Table VII), 4 (Table VIII) and 5 (Table IX) are shown. From device 3, path $c(3,1)$ is the optimum, for device 4 , path $f(4,2)$ and for device 5 , path $h, d(5,6,1)$. These results highlight the contrasting measurements used by the DCe-CAB in comparison to when (8) is used. Using this approach, the DCe-CAB again achieves a balance between attributes by selecting neither the maximum nor minimum values in these scenarios. The DCe-CAB is prepared however, to sacrifice a higher operational cost in the DC
Table VII. PATH SELECTION FROM DEVICE 3 USING (9)

\begin{tabular}{|c|c|c|c|c|c|c|c|c|c|}
\hline Client & \multicolumn{6}{|c|}{ Path to Data Centre } & \multirow{2}{*}{$\frac{\left(\left(x_{0}\right) \ldots(x),\left(, e n_{\infty}\right)\right.}{2}$} & \multirow{2}{*}{ 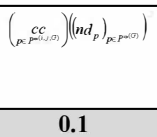 } & \multirow{2}{*}{$\begin{array}{l}\text { (9) } \\
0.2 \\
\end{array}$} \\
\hline 3 & 1 & - & - & - & - & - & & & \\
\hline 3 & 2 & - & - & - & - & - & 4 & 0.2 & 0.8 \\
\hline 3 & 1 & 6 & & - & - & - & 6 & 0.8 & 4.8 \\
\hline 3 & 2 & 1 & - & - & - & - & 2 & 0.6 & 1.2 \\
\hline 3 & 2 & 1 & 6 & - & - & - & 6 & 1.8 & 10.8 \\
\hline 3 & 2 & 1 & 6 & 7 & - & - & 3 & 3 & 9 \\
\hline 3 & 2 & 1 & 6 & 7 & 9 & - & 5 & 5 & 25 \\
\hline 3 & 2 & 1 & 6 & 7 & 9 & 12 & 10 & 9 & 90 \\
\hline
\end{tabular}

Table VIII. Path SELECTION From DeVice 4 USING (9)

\begin{tabular}{|c|c|c|c|c|c|c|c|c|c|}
\hline Client & \multicolumn{6}{|c|}{ Path to Data Centre } & \multirow{2}{*}{ 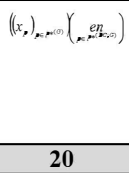 } & \multirow{2}{*}{ 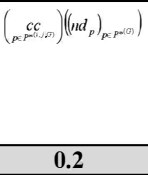 } & \multirow{2}{*}{$\begin{array}{l}\text { (9) } \\
\\
4\end{array}$} \\
\hline 4 & 2 & - & - & - & - & - & & & \\
\hline 4 & 2 & 1 & - & - & - & - & 10 & 0.6 & 6 \\
\hline 4 & 2 & 1 & 6 & - & - & - & 30 & 1.8 & 54 \\
\hline 4 & 2 & 1 & 6 & 7 & - & - & 15 & 3 & 45 \\
\hline 4 & 2 & 1 & 6 & 7 & 9 & - & 25 & 5 & 125 \\
\hline 4 & 2 & 1 & 6 & 7 & 9 & 12 & 50 & 9 & 450 \\
\hline
\end{tabular}

Table IX.

PATH SElECtion FROM DEVICE 5 USING (9)

\begin{tabular}{|c|c|c|c|c|c|c|c|}
\hline Client & \multicolumn{4}{|c|}{ Path to Data Centre } & \multirow{2}{*}{$\frac{\left(\left(x_{0}\right) \ldots \ldots(\ldots)\left(\ldots n_{\infty}\right)\right.}{15}$} & \multirow{2}{*}{ 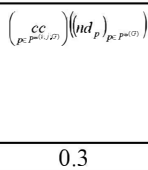 } & \multirow{2}{*}{$\begin{array}{l}\text { (9) } \\
\\
4.5 \\
\end{array}$} \\
\hline 5 & 6 & - & - & - & & & \\
\hline 5 & 6 & 1 & - & - & 5 & 0.8 & 4 \\
\hline 5 & 6 & 7 & - & - & 7.5 & 0.9 & 6.75 \\
\hline 5 & 6 & 1 & 2 & - & 10 & 1.8 & 18 \\
\hline 5 & 6 & 7 & 9 & - & 12.5 & 2.1 & 26.25 \\
\hline 5 & 6 & 7 & 9 & 12 & 25 & 4.8 & 120 \\
\hline
\end{tabular}

$\left(\left(x_{p}\right)_{p \in P *(G)}\left(\begin{array}{c}e n \\ p \in P *(D C ; G)\end{array}\right)\right.$ for a lower cost path between client and DC endpoints $(\underset{p \in P *(i, j)}{c \mathcal{C}})\left(\left(n d_{p}\right)_{p \in P^{*(G)}}\right)$. With regard to path selection for device 5 , for example, the minimum link cost (0.2) is selected, while the minimum DC cost (20) is not.

\section{3) Evaluation}

The DCe-CAB's balance between performance-influencing attributes and compromise on cost to optimise data centre communications can therefore be observed. While both approaches enable relationships between context attributes relating to the data centre, network environment and application characteristics to be balanced, average path cost between client device and DC is included by each. By incorporating characteristics on the end-to-end path into the decision-making process, it is possible to achieve a scenario where operational ability here is considered as important in the communication process as the performance characteristics within the data centre itself. A short path may be the most efficient in terms of propagation distance between communicating nodes, but it may be exposed to a higher carbon cost. In the case of device 4 in the case study for example, the shortest path is exposed to a less efficient operational environment and therefore higher average carbon cost than the path selected by the DCe-CAB. Through this work we therefore demonstrate how it is possible to optimise 
communication efficiency by including an extended range of context in the decision-making process.

Solutions presented in this paper are general in that they assume all context attributes in the model are populated, values remain constant, and the network topology remains static during the communication. With regard to assuming constant context values and a static topology, this generality will not exist in reality as the DCe-CAB collects context in real-time to allow changes to be incorporated in decisions made. In terms of the DCe-CAB's ability to cope when context is unavailable, there is a design assumption that substitute optimisation approaches will be used for which all context is present.

The model is strong in its design; despite being newly evolved with respect to the DC domain, it has grown from one optimised for operation in resource-constrained DTNs. This involved exploring the range of context collectable here and developing solutions which use the most useful context. In domains where resources are constrained or where the objective is to minimise energy consumption, restricting management overhead to that strictly necessary is a key design requirement to minimise network load, consumption of finite resources, and cost per transaction. A management approach may therefore appear simplistic when optimised to achieve the objectives for which it has been designed.

Optimisation approaches presented in this paper demonstrate strategies which prioritise carbon emission, electricity cost and waiting latency on the path to and within the data centre. It is not our intention to represent an assumption in these approaches that DC operators would optimise their network according to carbon emission as opposed to electricity cost. Equations (8) and (9) include carbon cost, but this element in the equation could be replaced with electrical cost or waiting latency. In a first pass experimentation with context evaluation to drive data centre and path selections, [15] presents a contrasting series of optimisations developed for the DC domain. These take into account characteristics of the operating environment only (including carbon, latency and electricity cost), and do not consider application characteristics nor the network's ability to support it in decisions made in contrast to approaches presented here. Work in this paper therefore represents the way in which the $\mathrm{DCe}-\mathrm{CAB}$ algorithm is evolving as ideas mature.

\section{CONCLUSION AND FURTHER WORK}

Energy-efficient management strategies are unique in data centres due to the scale of operation here. In this paper we demonstrate how approaches to energy challenges in DCs are standalone, tackling individual aspects such as optimising temperature or responding to platform type. Energy-managing frameworks provide an integrated approach, but do not consider the effects of paths traversed between clients and DCs. These facts contribute to a research gap for which we suggest a DCe-CAB algorithm which has scope to utilise a range of attributes and optimise the effectiveness of DC selection and operation. Furthermore, it integrates characteristics from the environment between client and DC into its decisions. This approach recognises that in the integration of energy-efficiency, there are cost overheads: in provisioning a single management solution for the DC domain, we strive to maximise the efficiency of energy-awareness.

As future work, we will extend the DC selection process to validate the DCe-CAB's ability to make optimised decisions using models presented in this paper and when using a wider range of attributes, including power effectiveness and CPU utilisation. The original implementation of the CAB for DTNs will simultaneously be extended to monitor energy-tolerant data centre networking using a test-bed deployment.

\section{ACKNOWLEDGEMENT}

We acknowledge Professor A. Jhunjhunwala and Dr. D. Jalihal from the Indian Institute of Technology, Madras for their contributions to this paper.

\section{REFERENCES}

[1] S. Ruth, "Green IT - More Than a Three Percent Solution?," IEEE Internet Computing, Vol. 13, Iss. 4, Jul./Aug. 2009, pp. 74-78.

[2] European Commission, "Code of Conduct on Data Centers Energy Efficiency," Oct. 2008, pp. 1-20.

[3] C. Guo, G. Lu, D. Li, H. Wu, X. Zhang, Y. Shi, C. Tian, Y. Zhang, and S. Lu, "BCube: A High Performance, Server-centric Network Architecture for Modular Data Centers," in Proc. of ACM SIGCOMM, Aug. 2009, pp. 63-74.

[4] C. Molloy and M. Iqbal, "Improving Data-Center Efficiency for a Smarter Planet," IBM Journal of Research and Development, Jul. 2010, pp. 1-8.

[5] Intel and T-Systems, "DataCenter 2020"; Available [Accessed: Feb. 2010]: http://www.datacenter2020.com.

[6] M. K. Patterson, "The Effect of Data Center Temperature on Energy Efficiency," in Proc. of $11^{\text {th }}$ IEEE Intersociety Conference on Thermal and Thermomechanical Phenomena in Electronic Systems, May 2008, pp. 1167-1174.

[7] R. Nathuji, C. Isci, and E. Gorbatov, "Exploiting Platform Heterogeneity for Power Efficient Data Centers," in Proc. of $4^{\text {th }}$ IEEE Int. Conf. on Autonomic Computing, Jun. 2007, pp. 5-15.

[8] L. Parolini, B. Sinopoli, and B. Krogh, "Reducing Data Center Energy Consumption via Coordinated Cooling and Load Management," in Proc. of ACM Workshop on Power Aware Computing and Systems, Dec. 2008, pp. 1-5.

[9] J. Quittek, R. Winter, T. Dietz, and D. Dudkowski, "Definition of Managed Objects for Energy Management," work in progress as an Internet Draft, Feb. 2010, pp. 1-46.

[10] B. Claise, M. Chandramouli, J. Parello, and B. Schoening, "Energy Monitoring MIB," work in progress as an Internet Draft, Mar. 2010.

[11] C. Guo, H. Wu, K. Tan, L. Shi, Y. Zhang, and S. Lu, "DCell: A Scalable and Fault-Tolerant Network Structure for Data Centers," in Proc. of ACM SIGCOMM, Aug. 2008, pp. 75-86.

[12] C. Peoples, G. Parr, B. Scotney, and A. Moore, "Context-Aware PolicyBased Framework for Self-Management in Delay-Tolerant Networks: A Case Study for Deep Space Exploration," IEEE Communications Magazine, Jul. 2010, Vol. 48, No. 7, pp. 102-109.

[13] Z. Dzalilov and I. Ouveysi, "Mathematical Models for Failure Reconfiguration of Telecommunication Networks: Part 1," Optimization 58, Dec. 2008, pp. 483-504.

[14] Department of Environment, Food and Rural Affairs (DEFRA), "BNXSO1: Carbon Dioxide Emission Factors for UK Energy Use," Mar. 2009, pp. 1-8.

[15] C. Peoples, G. Parr, and S. McClean, "Energy-Aware Data Centre Management," in Proc. of 17th National Conference of Communications, Jan. 2011, pp. 1-5. 